\title{
Schmerzarme Dauerdrainage
}

\section{Aszites-Behandlung bei Krebspatienten}

") Die Aszitesdrainage verbessert die Lebensqualität auch in den letzten Tagen. Damit wird die Selbstständigkeit des Patienten und seine Selbstbestimmung gefördert. Der Dauerdrainagekatheter lässt sich ambulant anlegen - ein insgesamt besehen risikoarmes Unterfangen.

Bei Dr. med. Thomas Wolff, Onkologie Lerchenfeld, Hamburg, stellt sich eine Patientin mit Ovarialkarzinom und Peritonealkarzinose vor. Sie wolle keine kausale Behandlung mehr, sondern lieber die palliative Versorgung. Vom Hausarzt erhielt sie wegen zunehmendem Bauchumfang ein wassertreibendes Medikament. Vier Monate später stellt sie sich zur ersten AszitesEntlastung in einer Klinik vor, weil der Bauch unter Spironolacton immer praller wurde. Dann kommt sie zu
Wolff in die Praxis. Er rät ihr zur Anlage einer Dauerdrainage, weil anzunehmen war, dass das Wasser immer wieder nachläuft. Wolff legte ihr ambulant die ASEPT ${ }^{\circledR}$-Drainage an. Weil die Patientin die ihr noch verbleibende Zeit zuhause verbringen möchte, organisierte Wolff ihr ein SAPV-Team und die nötigen Ablaufflaschen. Die Patientin konnte mit der Dauerdrainage zuhause sein, entlastete sich zweimal wöchentlich selbst und verstarb acht Wochen später an ihrer Grunderkrankung.

Auch palliative Therapiekonzepte können durch die Dauerdrainage begleitet werden. Dazu spritzt Wolff am Tag des Anlegens der ASEPT ${ }^{\circledR}$ Drainage Midazolam zur Lokalanästhesie. Er lässt zuerst nicht mehr als zwei Liter ab. Danach benötigt der $\mathrm{Pa}$ - tient keine spezielle Eiweißnahrung. Man forciere auch durch das Ablassen des Wassers nicht etwa ein Nachlaufen des Aszites oder die Ödem-Entstehung. Trotz des Drainage-Systems, das unter einem Folienverband liegt, lässt sich die Körperhygiene durchführen.

Der Vortrag ist als Film abrufbar: www.pfmmedical.com/medcare oder www.youtube.com/user/pfmmedical.

(namo)

Symposium im Rahmen der MEDCARE „Krebspatienten mit Aszites: Mehr Lebensqualität dank ambulanter Versorgung mit Drainage, individueller Ernährungstherapie und ganzheitlicher Betreuung"; Leipzig, 27.09.2017 (Veranstalter: pfm medical ag)

\section{Wundversorgung}

\section{Biofilm-Forschung erhält neue Impulse}

॥ Die Pflege von Patienten mit chronischen Wunden stellt hohe Anforderungen an das Können und die Belastbarkeit von Pflegekräften. Nicht selten finden sich auf und in solchen Wunden Biofilme, die es den Wundkeimen erlauben, sich sowohl dem körpereigenen Immunsystem durch eine Toleranzentwicklung als auch einer antibiotischen bzw. antimikrobiellen Behandlung durch die Ausbildung von Resistenzen weitgehend zu entziehen. Dies gelingt den Wundbakterien beispielsweise durch Matrix-Bildung oder Anpassung der Stoffwechsel-Aktivität, je nach Erfordernissen des jeweiligen Milieus. Folglich werden für eine Heilung wesentlich höhere Dosierungen an Antibiotika oder anderer antimikrobiell wirksamen Substanzen eingesetzt als in der Versorgung akuter Wunden. Nur so meinte man, eine Resistenzbildung verhindern zu können.

Der zweifelhafte Erfolg und die seit einiger Zeit geführte Debatte über den sinnvollen Einsatz von Antibiotika bestärkten die Motivation, sich in der klinischen Forschung nach anderen Lösun- gen umzusehen, wie Dr. Georgina Gethin, Head of School of Nursing and Midwifery der Universität Galway/Irland auseinandersetzte. Daher war es ein Anliegen der Forschung, die Rolle von Biofilmen in der Persistenz von Wundinfektionen zu erhellen. Ein Bau-

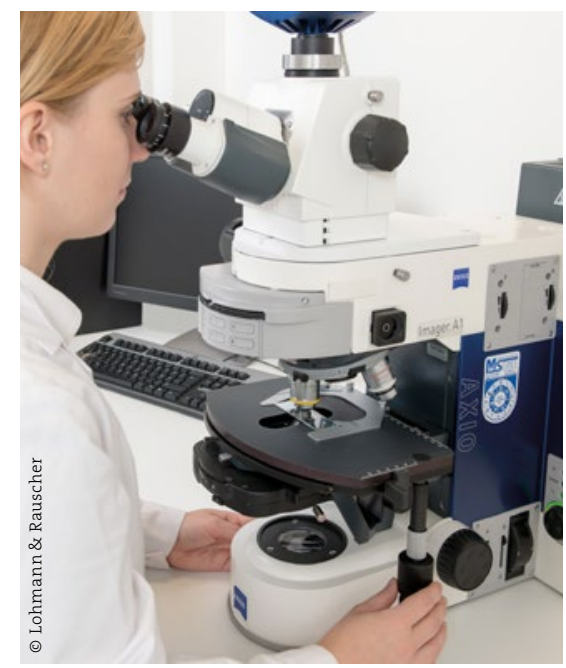

Für die Ausstattung der Labors wurden Geräte, z.B. ein Fluoreszenzmikroskop, angeschafft. stein dieser Forschung wird das erweiterte und neu konzipierte Mikrobiologie-Labor der Firma Lohmann \& Rauscher (L \& R) in Schönau bei Wien sein, das auf einem hochrangigen wissenschaftlichen Symposium vorgestellt wurde. Hier soll anhand von BiofilmModellen erforscht werden, wie weit die Behandlung mit antibakteriell wirksamen Wundauflagen (z.B. Suprasorb ${ }^{\circledR} \mathrm{X}$ plus $\mathrm{PHMB}$ ) optimiert werden kann. Zudem können klinisch relevante Bakterienstämme ausgetestet und so die Rolle von Biofilmen in Wunden realitätsnah dargestellt werden. Das Ziel dieser Forschung ist, die Interaktion von Keimen in Biofilmen, ihre Abwehrmechanismen gegen immunkompetente Zellen, aber auch gegen antimikrobiell aktive Substanzen, z.B. aus Wundauflagen, besser zu verstehen.

(tuk)

Scientific Spotlight „One step closer to manage biofilm in chronic wounds. $\mathrm{L}$ \& $\mathrm{R}$ biofilm research facility drives antimicrobial product technologies"; Wien, 25.10.2017 (Veranstalter: Lohmann \& Rauscher $\mathrm{GmbH}$ ) 